\title{
Dynamics of postoperative serum cortisol after transsphenoidal surgery for Cushing's disease: implications for immediate reoperation and remission
}

\author{
Marc Mayberg, MD, ${ }^{4}$ Stephen Reintjes, MD, ${ }^{3}$ Anika Patel, BS, ${ }^{1}$ Kelley Moloney, ARNP, ${ }^{1}$ \\ Jennifer Mercado, ARNP, ${ }^{1}$ Alex Carlson, BA, ${ }^{2}$ James Scanlan, PhD, ${ }^{2}$ and Frances Broyles, MD${ }^{1}$ \begin{abstract}
Neurological Surgery, University of Washington, Seattle, Washington; and ${ }^{3}$ Department of Neurological Surgery, University of South Florida, Tampa, Florida
\end{abstract} \\ ${ }^{1}$ Swedish Pituitary Center, Swedish Neuroscience Institute; ${ }^{2}$ Swedish Center for Research and Innovation; and ${ }^{4}$ Department of
}

OBJECTIVE Successful transsphenoidal surgery for adrenocorticotropin hormone (ACTH)-producing pituitary tumors is associated with subnormal postoperative serum cortisol levels, which may guide decisions regarding immediate reoperation. However, little is known about the detailed temporal course of changes in serum cortisol in the immediate postoperative period, and the relationship of postoperative cortisol dynamics to remission and late recurrence.

METHODS A single-center retrospective cohort analysis was performed for all patients undergoing pituitary surgery from 2007 through 2015. Standardized diagnostic and treatment algorithms were applied to all patients with potential Cushing's disease (CD), including microsurgical transsphenoidal adenomectomy (TSA) by a single surgeon. All patients had serum cortisol levels drawn at 6 -hour intervals for 72 hours after surgery, and were offered reoperation within 3 days for normal or supranormal postoperative cortisol levels. Primary outcomes were 6-month remission and late recurrence; secondary outcomes were persistent postoperative hypocortisolism and surgical morbidity. Discriminatory levels of postoperative serum cortisol for predicting remission were calculated at various intervals after surgery using receiver operating characteristic (ROC) curves.

RESULTS Among 89 patients diagnosed with CD, 81 underwent initial TSA for a potentially curable lesion; 23 patients (25.8\%) underwent an immediate second TSA. For the entire cohort, 6-month remission was achieved in $77.8 \%$ and late recurrences occurred in $9.5 \%$, at a mean of 43.5 months. Compared with patients with a single surgery, those with an immediate second TSA had similar rates of remission ( $78.3 \%$ vs $77.6 \%)$ and late recurrence $(5.6 \%$ vs $11.1 \%)$. The rate of hypocortisolism for patients with 2 surgeries $(12 / 23,52.2 \%)$ was significantly greater than that for patients with single surgeries $(13 / 58,22.4 \% ; p<0.001)$. There was no difference in the incidence of CSF leaks between the first and second operations. Remission was achieved in $58(92.1 \%)$ of 64 patients who completed the 2-surgery protocol. The temporal course of postoperative serum cortisol levels among patients varied considerably, with subnormal nadir levels $<2 \mu \mathrm{g} / \mathrm{dl}$ occurring between 12 hours and 66 hours. Patients achieving remission had significantly lower mean serum cortisol levels at every time point after surgery $(p<0.01)$. By ROC curve analysis, nadir cortisol levels $<2.1 \mu \mathrm{g} / \mathrm{dl}$ were predictive of 6 -month remission for the entire cohort over 3 days (positive predictive value [PPV] $=94 \%$ ); discriminating cortisol levels for predicting remission on postoperative day (POD) 2 were $<5.4 \mu \mathrm{g} / \mathrm{dl}$ (PPV = 97\%), although patients with remission after postoperative cortisol levels of 2-5 $\mu \mathrm{g} / \mathrm{dl}$ had a significantly higher rate of late recurrence.

CONCLUSIONS There is substantial variation in the temporal course of serum cortisol levels over the first 72 hours after TSA for CD, with nadir levels predictive for remission occurring as late as POD 3. Although a cortisol level of $2.1 \mu \mathrm{g} /$ $\mathrm{dl}$ at any point was an accurate predictor of 6 -month remission, levels less than $5.4 \mu \mathrm{g} / \mathrm{dl}$ on POD 2 were reasonably accurate. These data may enable decisions regarding the efficacy of an immediate second surgical procedure performed during the same hospitalization; immediate reoperation is associated with excellent remission rates and low recurrence rates in patients otherwise unlikely to achieve remission, but carries a higher risk of permanent hypocortisolism. https://thejns.org/doi/abs/10.3171/2017.6.JNS17635

KEY WORDS Cushing's disease; transsphenoidal surgery; postoperative cortisol; reoperation; remission; late recurrence; pituitary surgery

ABBREVIATIONS $\mathrm{ACTH}=$ adrenocorticotropin hormone; $\mathrm{AUC}=$ area under the curve; $\mathrm{BIPSS}=$ bilateral inferior petrosal sinus sampling; $\mathrm{BMI}=$ body mass index; $\mathrm{CD}=$ Cushing's disease; $\mathrm{CST}=$ cosyntropin stimulation test; NPV = negative predictive value; PACS = picture archiving and communication system; POD = postoperative day; PPV = positive predictive value; ROC = receiver operating characteristic; TSA = transsphenoidal adenomectomy; UFC = urinary free cortisol.

SUBMITTED March 15, 2017. ACCEPTED June 5, 2017.

INCLUDE WHEN CITING Published online December 22, 2017; DOI: 10.3171/2017.6.JNS17635. 
$\mathrm{R}$ ESECTION by transsphenoidal adenomectomy (TSA) remains the treatment of choice for Cushing's disease (CD) due to adrenocorticotropin hormone $\left(\right.$ ACTH)-secreting pituitary adenomas. ${ }^{18}$ More than $90 \%$ of patients with CD harbor pituitary microadenomas, ${ }^{10}$ and numerous reports have described postoperative remission rates of $60 \%-90 \%, 7,12,19,32,33$ with late recurrence in $10 \%$ $65 \%$ of patients. ${ }^{2,4,5}$ Factors associated with unsuccessful surgery for CD include larger tumor size, ${ }^{13,27}$ absence of tumor on MRI, ${ }^{11,31}$ previous transsphenoidal surgery, ${ }^{9,29}$ and invasion of the dura ${ }^{17}$ and/or cavernous sinus. ${ }^{6,27}$

Successful transsphenoidal surgery for CD is usually associated with transient hypocortisolism lasting 3-12 months, likely due to long-standing suppression of corticotrophs in the normal pituitary gland by persistently elevated serum cortisol and ACTH in CD. ${ }^{3,20,23,26}$ Postoperative hypocortisolism is manifest within the first days of surgery, and subnormal levels of serum cortisol nadir at various times in the postoperative period have been associated with subsequent remission. ${ }^{1,12,26}$ In this regard, the measurement of postoperative cortisol levels shortly after surgery for $\mathrm{CD}$ has become a widely used measure to assess remission of $\mathrm{CD}$ in the early postoperative period; several reports have reported a high positive predictive value (PPV) for remission if the serum cortisol level is < $5 \mu \mathrm{g} / \mathrm{dl}$ within $2-7$ days of adenomectomy. ${ }^{16,22,28,30,34}$ Additionally, early repeat surgery for persistently high cortisol levels in the immediate postoperative period has been advocated as a means to increase the percentage of surgical patients with $\mathrm{CD}$ achieving remission. ${ }^{15,24}$ With the objective of offering an immediate second surgery to patients otherwise unlikely to achieve remission, it is important to know the optimal timing and most accurate discriminating value from postoperative cortisol determinations to predict remission. However, many studies examining the significance of postoperative cortisol levels after TSA included patients who received supplemental corticosteroids in the perioperative period, did not correlate the level and timing of postoperative cortisol nadir with late recurrence, and measured cortisol at only 1 or 2 time points after surgery. ${ }^{21}$ Little is known about the dynamic changes in serum cortisol in the first 72 hours of the postoperative period, as well as the relationship between the timing and degree of these changes and remission. This is particularly important in the decision of whether to consider immediate second surgery, which is optimally performed before postoperative inflammation and fibrosis may impede the identification of any remaining tumor remnant.

Since 2007, all patients with CD at our institution have been treated according to a standardized diagnostic and therapeutic protocol that includes uniform criteria for the diagnosis of $\mathrm{CD},{ }^{18}$ surgical treatment by TSA (Fig. 1), withholding perioperative corticosteroids, drawing serum cortisol levels every 6 hours for the first 72 hours after surgery, and recommending an immediate second surgery when postoperative serum cortisol is believed to be reflective of residual tumor. We retrospectively analyzed postoperative cortisol levels in 89 consecutive patients undergoing TSA for CD to determine 1) the dynamic temporal variations in cortisol levels over 72 hours, 2) the relationship of preoperative clinical and imaging variables to variations in postoperative cortisol level dynamics, 3) the relationship of dynamic patterns in postoperative serum cortisol to remission and late recurrence, 4) the optimum cortisol values predicting remission on postoperative days (PODs) 1-3, and 5) the safety and efficacy of immediate second surgery on remission rates and late recurrence.

\section{Methods \\ Study Design}

This study was a retrospective single-center cohort analysis derived from an IRB-approved prospective database of all patients undergoing pituitary surgery at the Swedish Neuroscience Institute from 2007 through 2015. Additional IRB approval was obtained to investigate the temporal dynamics of immediate postoperative cortisol in patients undergoing TSA for CD. Patient data were extracted from electronic medical records and an MRI picture archiving and communication system (PACS).

\section{Diagnosis of Patients With CD}

All patients with the presumptive diagnosis of $\mathrm{CD}$ were evaluated and treated according to established protocols. ${ }^{18}$ Diagnostic criteria for CD included history and physical examination by a neuroendocrinologist, and a series of diagnostic endocrine tests, including multiple 24-hour urinary free cortisol (UFC) levels, elevated random plasma ACTH and cortisol levels, nonsuppressible plasma cortisol $(<5 \mu \mathrm{g} / \mathrm{dl})$ with low-dose dexamethasone, and disruption of the diurnal adrenocortical secretion pattern reflected by elevated midnight serum and/or salivary cortisol. Patients underwent MRI evaluation of the pituitary gland before surgery using standardized protocols, including triaxial contrast-enhanced MRI of the sella and parasellar region with dynamic contrast sequences. All patients, except those patients with previous surgery documenting ACTHproducing tumor or patients refusing the test, underwent bilateral inferior petrosal sinus sampling (BIPSS) with corticotropin-releasing hormone stimulation to confirm a central source of ACTH, with an inferior petrosal sinus/ peripheral venous ratio of 3 considered positive. Following consensus diagnosis of CD by a multidisciplinary pituitary team, patients were evaluated for TSA.

\section{Surgical and Perioperative Management of Patients With CD}

All patients underwent endoscope-assisted microscopic TSA by the first author (M.M.) using stereotactic navigation and surgical protocols (Fig. 1). The side of the initial exploration of the pituitary gland was based upon MRI and/or BIPSS findings, and the contralateral anterior lobe and central portion of the gland were carefully incised and explored in all cases. Capsulectomy and en bloc resection were attempted for all microadenomas, whereas macroadenomas were removed in a piecemeal fashion. If the exploration on both sides of the gland at the initial surgery was negative, hemihypophysectomy on the side of the BIPSS localization was performed. In all first operations, a less aggressive resection was employed to preserve sufficient gland to maintain pituitary function. No exogenous corticosteroids were administered during 
surgery or in the immediate postoperative period. Surgical complications were recorded, including death, vascular injury, vision loss, diplopia, neurological injury, or any CSF leak requiring lumbar drain or reoperation. After surgery, patients remained hospitalized and serum cortisol levels were tested every 6 hours beginning after completion of surgery (time $=0$ ) until 72 hours. Samples were withdrawn from a central venous catheter to reduce stress associated with venipuncture, and cortisol results were readily available within 1-2 hours after sampling. Presumed remission was defined a priori as serum cortisol $<2 \mu \mathrm{g} /$ $\mathrm{dl}$ on 2 successive determinations 6 hours apart, in which case the patient was started on hydrocortisone and discharged home. If the cortisol was persistently more than 5 $\mu \mathrm{g} / \mathrm{dl}$ after 72 hours, an immediate repeat TSA during the same hospitalization was recommended unless there was a history of prior TSA for CD. For patients with cortisol nadir levels between 2 and $5 \mu \mathrm{g} / \mathrm{dl}$, the decision regarding second surgery was based on patient preference on the basis of age and endocrine status (e.g., fertility). All second TSA procedures were performed within 5 days of the initial surgery, with more than $90 \%$ completed within 3 days. For cases in which tumor was encountered at the initial surgery, at the second surgery a wider excision of the gland at that site and resection of the adjacent dura were performed (Fig. 1). If the initial surgery did not reveal tumor, bilateral partial hemihypophysectomies with multiple incisions into the remaining gland were performed with an attempt to preserve a small amount of functional tissue adjacent to the stalk.

\section{Postdischarge Evaluation and Management}

Patients with 72-hour postoperative cortisol levels $<2$ $\mu \mathrm{g} / \mathrm{dl}$ were maintained on oral hydrocortisone as outpatients, with the dose gradually tapered according to symptoms and documentation of recovery of the pituitary-adrenal axis using the cosyntropin stimulation test (CST). Patients with an abnormal CST at 12 months were considered permanently hypocortisolemic and maintained on oral corticosteroids. A full neuroendocrine evaluation was conducted at 6 weeks, 6 months, and annually thereafter. All patients with apparent remission had 24-hour UFC testing and MRI at 6 months after surgery and annually thereafter. Patients without remission were offered the options of stereotactic radiosurgery, medical therapy (ketoconazole or pasireotide), and/or bilateral adrenalectomy. These patients were recorded as surgical nonremission, and any subsequent responses to adjunctive therapies were not included in the outcome analyses. Patients with initial remission and late recurrence were considered for either repeat surgery or alternative treatments as above, and similarly censored from further outcome analysis.

\section{Pituitary Surgery Database}

Patient data were extracted from electronic medical records and an MRI PACS into an IRB-approved prospective database maintained since 2005 , which included demographic and clinical features (age, sex, body mass index [BMI], and serum glucose and insulin requirements), diagnostic laboratory studies (full endocrine panel, dexamethasone suppression, UFC, BIPSS, and midnight serum and/or salivary cortisol), and imaging data (see below). Inpatient hospital data included surgical findings with laterality of the tumor, postoperative cortisol values at 6-hour intervals, and the occurrence of surgical morbidity as above.

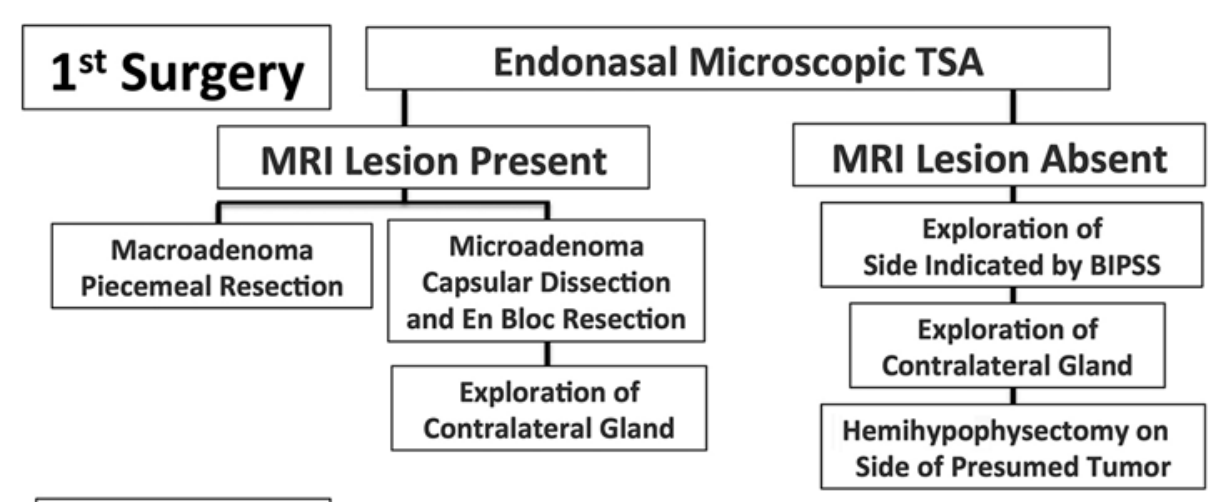

\section{$2^{\text {nd }}$ Surgery}

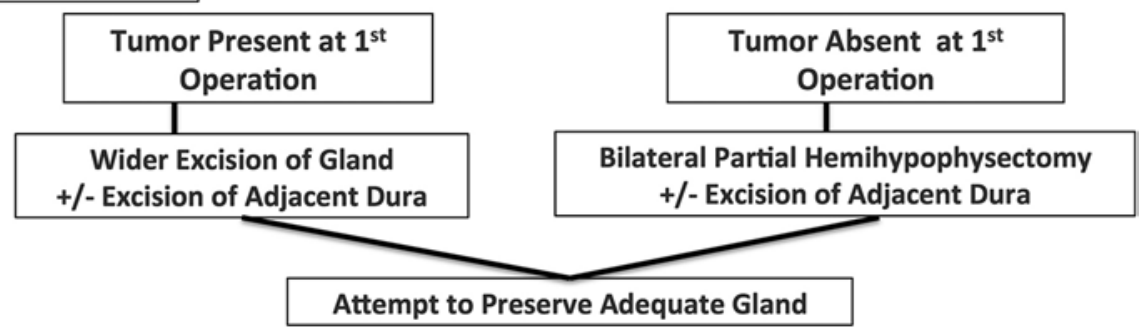

FIG. 1. Schematic diagram showing the surgical algorithm for pituitary exploration and tumor resection in initial and immediate second TSA for CD (see text for details). 


\section{Imaging Data}

All patients underwent preoperative pituitary-specific triaxial contrast-enhanced MRI, including dynamic contrast scans of the pituitary gland. For the current study, each MR image was blindly reassessed by an experienced neuroradiologist for greatest diameter of tumor, side of tumor, and cavernous sinus involvement, including Knosp scale grade. ${ }^{14}$

\section{Outcomes}

Primary outcomes were 6-month remission (normal 24-hour UFC and normal random serum cortisol at 6 months) and late recurrence (diagnosis of CD by the diagnostic protocol described above after documented initial remission at 6 months). Secondary outcomes included any surgical mortality or significant morbidity (vascular injury, vision loss, diplopia, neurological injury, cardiopulmonary complication, or any CSF leak requiring lumbar drain or reoperation) and hypocortisolism (low morning fasting cortisol and abnormal CST) at 12 months after surgery or later. New postoperative hypopituitarism involving pituitary hormones other than ACTH was not assessed in this analysis. The duration of follow-up was defined as the interval in months from date of surgery to last recorded pituitary center assessment.

\section{Statistical Analysis}

Univariate comparison of preoperative demographic, clinical, and imaging variables was performed using ttests for continuous variables and chi-square for ordinal or nominal variables. Logistic regressions using forward conditional variable entry were used to predict 6-month remission and late recurrence for 3 cohorts defined by postoperative cortisol level $<2.1 \mu \mathrm{g} / \mathrm{dl}, 2.1-5.4 \mu \mathrm{g} / \mathrm{dl}$, and $>$ $5.4 \mu \mathrm{g} / \mathrm{dl}$. Receiver operating characteristic (ROC) curves comparing lowest postoperative cortisol versus 6-month remission were constructed for nadir cortisol values on PODs 1 and 2, and for the entire cohort at all time points. Area under the curve (AUC) and Youden's Index were used to determine the most accurate discriminating cortisol value for predicting remission in each group, expressed as sensitivity, specificity, PPV, and negative predictive value (NPV). Statistical analyses were performed using IBM SPSS (version 19, IBM Corp.).

\section{Results}

\section{Patient Cohort}

The characteristics of the patient cohort are shown in Tables 1 and 2. The patient cohort demographics were typical for $\mathrm{CD}$, with a female preponderance $(83 \%)$, relatively young age (mean 38.1 years), and a high prevalence of obesity (mean BMI $35.4 \mathrm{~kg} / \mathrm{m}^{2}$ ) and insulin-dependent diabetes (17.3\%). Tables 1 and 2 show the distribution of patients as the postoperative cortisol monitoring protocol was applied to 89 consecutive patients with a presumptive diagnosis of $\mathrm{CD}$ who underwent 111 TSA procedures during the interval of 2007-2015. Four additional patients with $\mathrm{CD}$ underwent surgery during this interval by a surgeon not using this protocol, and were excluded from
TABLE 1. Clinical factors associated with remission and late recurrence

\begin{tabular}{|c|c|c|c|c|c|c|}
\hline Clinical Factor & $\mathrm{n}$ & $\begin{array}{c}\text { Remission } \\
(\%)\end{array}$ & $\begin{array}{c}p \\
\text { Value }\end{array}$ & $\mathrm{n}$ & $\begin{array}{l}\text { Recurrence } \\
(\%)\end{array}$ & $\begin{array}{c}p \\
\text { Value }\end{array}$ \\
\hline Sex & & & 0.9374 & & & 0.2816 \\
\hline Male & 14 & $11(79 \%)$ & & 11 & $2(18)$ & \\
\hline Female & 67 & $52(78 \%)$ & & 52 & $4(8)$ & \\
\hline Surgery epoch & & & 0.315 & & & 0.188 \\
\hline 2007-2009 & 30 & $25(83)$ & & 25 & $4(16)$ & \\
\hline 2010-2012 & 39 & $28(72)$ & & 28 & $1(4)$ & \\
\hline 2013-2015 & 12 & $10(83)$ & & 9 & $1(11)$ & \\
\hline Prior surgery & & & 0.0003 & & & 0.161 \\
\hline No & 61 & $54(88)$ & & 54 & $4(7)$ & \\
\hline Yes & 20 & $9(45)$ & & 9 & $2(22)$ & \\
\hline BIPSS side* & & & 0.574 & & & 0.521 \\
\hline Rt & 29 & $25(86)$ & & 25 & $4(16)$ & \\
\hline $\mathrm{Lt}$ & 18 & $15(83)$ & & 15 & $1(7)$ & \\
\hline Bilat & 6 & $6(100)$ & & 6 & $1(17)$ & \\
\hline Insulin use & & & 0.145 & & & 0.252 \\
\hline No & 67 & $54(81)$ & & 54 & $7(13)$ & \\
\hline Yes & 14 & $9(64)$ & & 9 & $0(0.0)$ & \\
\hline $\begin{array}{l}\text { MRI tumor size } \\
\quad(\mathrm{mm})\end{array}$ & & & 0.146 & & & 0.551 \\
\hline Not visible & 36 & $28(78)$ & & 28 & $2(7)$ & \\
\hline $1-10$ & 42 & 34 (81) & & 34 & $4(12)$ & \\
\hline$>10$ & 3 & $1(33)$ & & 1 & $0(0.0)$ & \\
\hline MRI side* & & & 0.238 & & & 0.114 \\
\hline Rt & 24 & $20(83)$ & & 20 & $6(30)$ & \\
\hline $\mathrm{Lt}$ & 15 & $12(80)$ & & 12 & $0(0.0)$ & \\
\hline Bilat & 6 & $3(50)$ & & 3 & $0(0.0)$ & \\
\hline Knosp grade & & & 0.775 & & & 0.510 \\
\hline 0 & 75 & $58(77)$ & & 58 & $6(10)$ & \\
\hline 1 & 6 & $5(83)$ & & 5 & $1(20)$ & \\
\hline Surgery side* & & & 0.599 & & & 0.651 \\
\hline Rt & 41 & $31(76)$ & & 31 & $5(16)$ & \\
\hline $\mathrm{Lt}$ & 25 & $20(80)$ & & 20 & $1(5)$ & \\
\hline Bilat & 10 & $9(90)$ & & 9 & 1 (11.1) & \\
\hline
\end{tabular}

Boldface type indicates statistical significance.

* Not measured in every patient.

analysis. Five patients who were treated according to the $\mathrm{CD}$ protocol were excluded when immunohistochemical analysis of the tumor specimen showed adenoma without ACTH staining. Three patients had noncurable tumors due to Knosp Grade 3 or higher on MRI scans. The total number of potentially curable patients was 81 , who underwent 104 operations (81 first operations and 23 second operations). The postoperative cortisol monitoring protocol was strictly followed in all cases with only minor exceptions. Seventeen patients with postoperative cortisol $>2$ $\mu \mathrm{g} / \mathrm{dl}$ after initial surgery did not undergo second surgery for the following reasons: 1) postoperative cortisol $>5 \mu \mathrm{g}$ / $\mathrm{dl}$ with history of previous TSA for CD $(\mathrm{n}=11)$, and 2$)$ postoperative cortisol $2-5 \mu \mathrm{g} / \mathrm{dl}$ with patient deferral of 
TABLE 2. Additional clinical factors associated with remission and late recurrence

\begin{tabular}{|c|c|c|c|c|c|c|c|c|c|c|}
\hline \multirow[b]{3}{*}{ Clinical Factor } & \multicolumn{4}{|c|}{ Remission } & \multirow[b]{3}{*}{ p Value } & \multicolumn{4}{|c|}{ Late Recurrence } & \multirow[b]{3}{*}{$p$ Value } \\
\hline & \multicolumn{2}{|c|}{ Yes } & \multicolumn{2}{|c|}{ No } & & \multicolumn{2}{|c|}{ Yes } & \multicolumn{2}{|c|}{ No } & \\
\hline & Mean & SD & Mean & SD & & Mean & SD & Mean & SD & \\
\hline Age (yrs) & 36.2 & 12.7 & 40.7 & 20.6 & 0.400 & 31.1 & 14.4 & 36.8 & 12.5 & 0.270 \\
\hline $\mathrm{BMI}\left(\mathrm{kg} / \mathrm{m}^{2}\right)$ & 33.9 & 8.4 & 36.8 & 12.8 & 0.254 & 31.5 & 6.9 & 34.2 & 8.6 & 0.433 \\
\hline 24-hr UFC ( $\mu \mathrm{g} / 24$ hrs) & 158.1 & 117.4 & 254.4 & 300.8 & 0.227 & 211.9 & 107.8 & 150.4 & 117.7 & 0.198 \\
\hline Midnight cortisol (ng/dl) & 31.3 & 44.9 & 26.0 & 12.8 & 0.665 & 27.1 & 22.5 & 31.8 & 46.8 & 0.827 \\
\hline Serum glucose (mg/dl) & 106.7 & 45.7 & 101.0 & 36.7 & 0.639 & 96.4 & 17.3 & 107.6 & 47.4 & 0.604 \\
\hline
\end{tabular}

second surgery because of concerns regarding hypopituitarism $(n=6)$. Five of these 17 patients achieved 6-month remission (Fig. 2).

\section{Primary Outcomes}

Table 3 and Fig. 2 show primary and secondary outcomes after TSA, and the comparative outcomes for patients who underwent 1 versus 2 surgeries. The overall 6-month remission for all potentially curable patients with CD was 63/81 (77.8\%). At a mean follow-up of 49.3 months (range 9-109 months), late recurrences occurred in $6(9.5 \%)$ of 63 patients at a mean of 43.5 months (range 1863 months). Compared with patients with a single surgery, those with an immediate second TSA had similar rates of remission $(78.3 \%$ vs $77.6 \%, \mathrm{p}=0.784)$ and late recurrence (5.6\% vs $11.1 \%, \mathrm{p}=0.541)$. Figure 2 shows the distribution

\section{Diagnosis of Cushing's Disease 89}
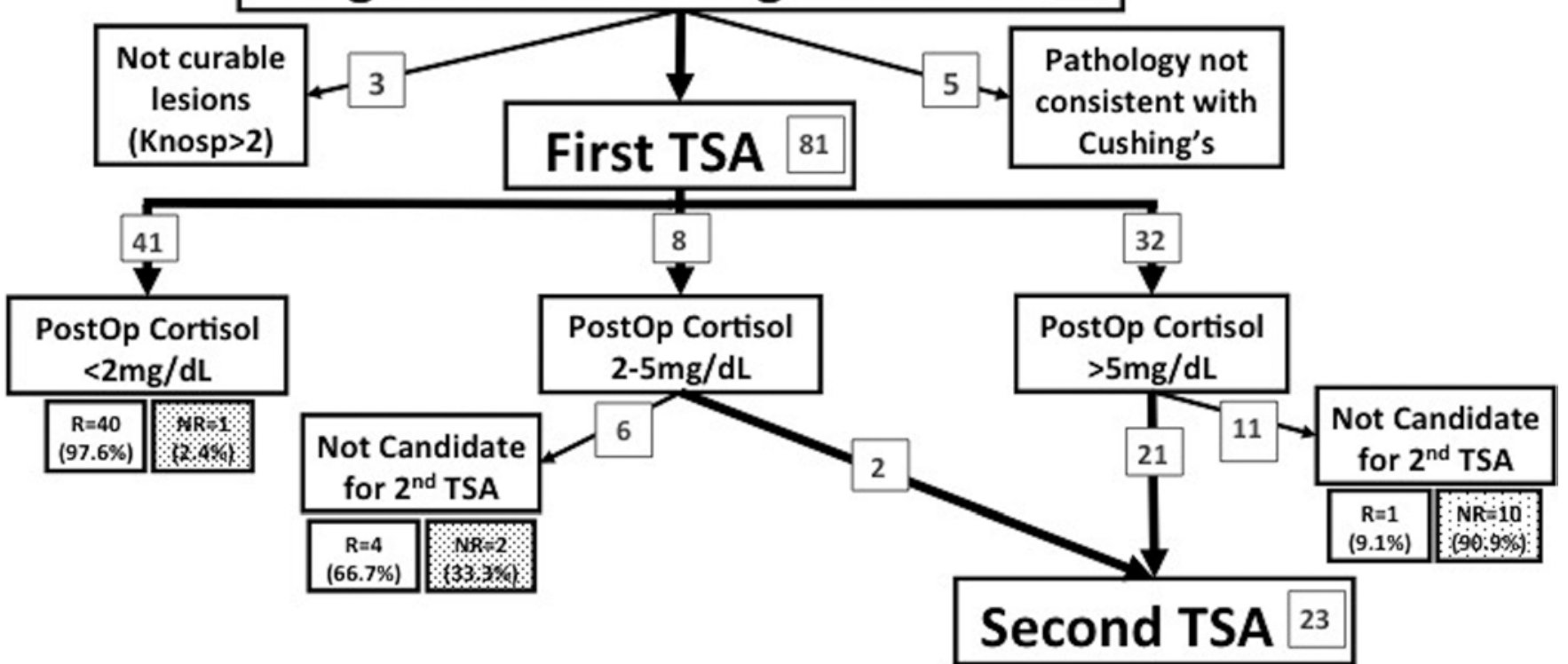

\begin{tabular}{|c|c|c|c|c|c|c|}
\cline { 2 - 7 } \multicolumn{1}{c|}{} & \multicolumn{5}{c|}{ Post-Operative Cortisol Nadir } \\
\cline { 2 - 7 } \multicolumn{1}{c|}{} & \multicolumn{2}{c|}{$<\mathrm{mg} / \mathrm{dL}$} & \multicolumn{2}{|c|}{$2-5 \mathrm{mg} / \mathrm{dL}$} & \multicolumn{2}{c|}{$>5 \mathrm{mg} / \mathrm{dL}$} \\
\cline { 2 - 7 } & $\mathrm{N}$ & Remission & $\mathrm{N}$ & Remission & $\mathrm{N}$ & Remission \\
\hline $\begin{array}{c}\text { Single } \\
\text { Surgery }\end{array}$ & 41 & $40(97.6 \%)$ & $6 *$ & $4(66.7 \%)$ & $11^{*}$ & $1(9.1 \%)$ \\
\hline $\begin{array}{c}\text { Two } \\
\text { Surgeries }\end{array}$ & 13 & $12(92.3 \%)$ & 6 & $5(83.3 \%)$ & 4 & $1(25 \%)$ \\
\hline Total & 54 & $52(96.2 \%)$ & 12 & $9(75 \%)$ & 15 & $2(13.3 \%)$ \\
\hline
\end{tabular}

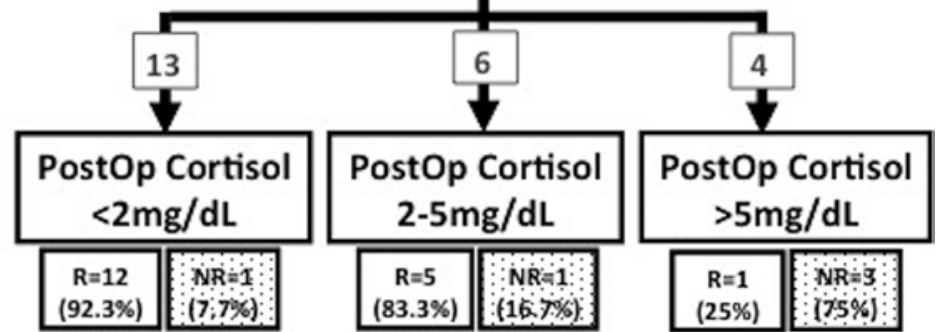

FIG. 2. Schematic diagram showing distribution and remission rates of subgroups based on postoperative cortisol measurements and immediate second surgery (see text for details). Calculations of rates for remission (R, white box) and nonremission (NR, hatched box) are shown below each subgroup. 
TABLE 3. Comparison of outcomes for initial versus second surgery

\begin{tabular}{lcccc}
\hline \multicolumn{1}{c}{ Outcome } & $\begin{array}{c}\text { Initial } \\
\text { Surgery (\%) }\end{array}$ & $\begin{array}{c}\text { 2nd } \\
\text { Surgery (\%) }\end{array}$ & $\begin{array}{c}\text { Total (\%) } \\
\text { Value }\end{array}$ \\
\hline No. of patients & 58 & 23 & 81 & \\
\hline Remissions & $45(77.6)$ & $18(78.3)$ & $63(77.8)$ & 0.784 \\
\hline Late recurrences & $5 / 45(11.1)$ & $1 / 18(5.6)$ & $6 / 63(9.5)$ & 0.541 \\
\hline CSF leaks & $11 / 81(13.6)$ & $3 / 23(13.0)$ & $14 / 104(13.5)$ & 0.947 \\
\hline Hypocortisolism & $13(22.4)$ & $12(52.2)$ & $25(30.8)$ & $\mathbf{0 . 0 0 0 8}$ \\
\hline
\end{tabular}

Boldface type indicates statistical significance.

of patients and remission rates according to the surgical algorithm. In the cohort of patients for whom second TSA was performed when initial surgery postoperative cortisol was $>2 \mu \mathrm{g} / \mathrm{dl}$, remissions were achieved in 40/41 for single surgery and in 18/23 for 2 surgeries $(58 / 64,92.1 \%)$. For 54 patients achieving a postoperative cortisol level $<2 \mu \mathrm{g} /$ $\mathrm{dl}$, the overall remission rate was $96.2 \%$, including both single-surgery (97.6\%) and 2-surgery (92.3\%) cohorts. The remission rate was $75 \%$ in 12 patients with postoperative cortisol levels of $2-5 \mu \mathrm{g} / \mathrm{dl}$, and $13.3 \%$ in patients with postoperative cortisol levels of $>5 \mu \mathrm{g} / \mathrm{dl}$.

\section{Secondary Outcomes}

There were no deaths or significant surgical morbidity among the overall cohort. Table 3 shows the frequency of CSF leaks and hypocortisolism for patients with 1 or 2 operations. For all patients, a CSF leak requiring a lumbar drain or reexploration occurred in $14(13.5 \%)$ of 104 operations. There was no significant difference in the prevalence of CSF leak in initial operations compared with second operations $(\mathrm{p}=0.947)$. In the overall cohort, persistent hypocortisolism occurred in 25 (30.9\%) of 81 patients. The rate of hypocortisolism for patients with 2 surgeries $(12 / 23,52.2 \%)$ was significantly greater than that for patients with single surgeries $(13 / 58,22.4 \%$; $\mathrm{p}<0.001)$.

\section{Clinical and Imaging Variables Related to Outcomes}

By univariate analysis the only significant association between demographic or imaging parameters and 6-month remission or late recurrence was history of prior surgery (Table 1). Compared with patients with no prior surgery for $C D(n=61), 20$ patients with a history of previous surgery had significantly lower 6-month remission rates $(45 \%$ vs $88 \%, p=0.0003$ ), and a suggestion of higher late recurrence rates $(22 \%$ vs $7 \%, \mathrm{p}=0.161)$. There was no association with primary outcome measures observed for any other preoperative baseline clinical characteristic or diagnostic test, including age, sex, BMI, preoperative 24-hour UFC level, salivary cortisol, tumor site, or glucose or insulin requirement. According to MRI data, tumor size was not significantly associated with remission, recurrence, or need for second operation, although only 1 of 3 patients with a tumor diameter $>10 \mathrm{~mm}$ achieved remission.

\section{Dynamics of Postoperative Serum Cortisol}

Figure 3 shows the mean postoperative serum cortisol measurements at 6-hour intervals in patients achieving

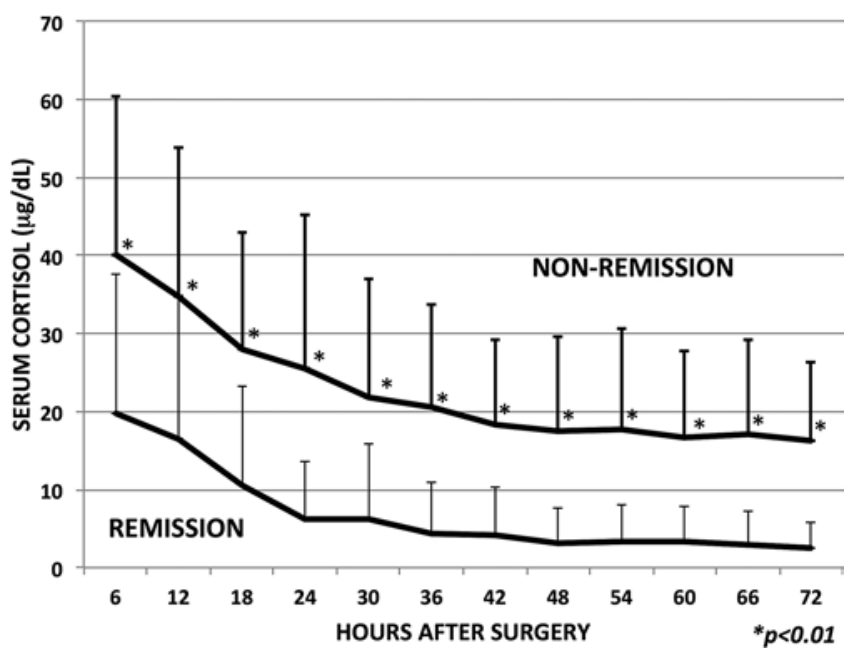

FIG. 3. Mean serum cortisol measurements at 6 -hour intervals after surgery for $\mathrm{CD}$ for patients achieving remission (lower line) versus those without remission (upper line). Values are expressed as means \pm SDs. There were significant differences in cortisol levels between groups at each time interval $(p<0.01)$.

6-month remission versus those not achieving remission. There was a significant difference in mean serum cortisol level at every time point after surgery from 6 to 72 hours $(\mathrm{p}<0.01)$. Both groups showed higher cortisol levels immediately after surgery, although a significant difference in cortisol between groups was apparent within 6 hours. Cortisol levels in both groups declined rapidly within the first 24 hours to reach a relatively stable level by $36-72$ hours. However, there was substantial variation among individual patient cortisol time curves, as shown in Fig. 4. The majority of patients in remission showed declines in cortisol to $<2 \mu \mathrm{g} / \mathrm{dl}$ within 36 hours, although several patients did not reach this level for 48-72 hours, with the latest nadir $<2 \mu \mathrm{g} / \mathrm{dl}$ noted at 66 hours after surgery. Patients in both the remission and nonremission groups showed occasional transient spike elevations in cortisol followed by subsequent continuing decline (Fig. 4, patients B and C).

To determine the best postoperative cortisol nadir value and timing to predict 6-month remission, ROC curves were constructed for cortisol values on PODs 1 and 2 and the cumulative data set (Table 4). As reflected by the AUC value, the accuracy of the serum cortisol test in predicting remission was good on POD $1(\mathrm{AUC}=0.831)$ and excellent on POD 2 (AUC $=0.917)$ and for the entire cohort (AUC $=0.922$ ). For the entire cohort over 72 hours, the optimal postoperative cortisol value predicting remission was 2.14 $\mu \mathrm{g} / \mathrm{dl}$ (sensitivity 0.825 , specificity 0.812 ). However, reasonably equivalent accuracy was noted for levels of 5.29 $\mu \mathrm{g} / \mathrm{dl}$ on POD 1 (sensitivity 0.730 , specificity 0.875 ) and $5.35 \mu \mathrm{g} / \mathrm{dl}$ on POD 2 (sensitivity 0.844 , specificity 0.933 ). The highest PPV and NPV were observed for the POD 2 value of $5.35 \mu \mathrm{g} / \mathrm{dl}$ (97\% and 73\%, respectively). When these cortisol discrimination levels were applied post hoc to all 81 potentially curable patients with $\mathrm{CD}$ (Table 5), 59 achieved serum cortisol nadirs $<2.1 \mu \mathrm{g} / \mathrm{dl}$ within 72 hours; of these, remission was achieved in $52(88.1 \%) ; 29$ of 30 on POD 1, 17 of 18 on POD 2, and 6 of 6 on POD 3. For patients with nadir cortisol values in the range of 2.1-5.4 $\mu \mathrm{g} /$ 


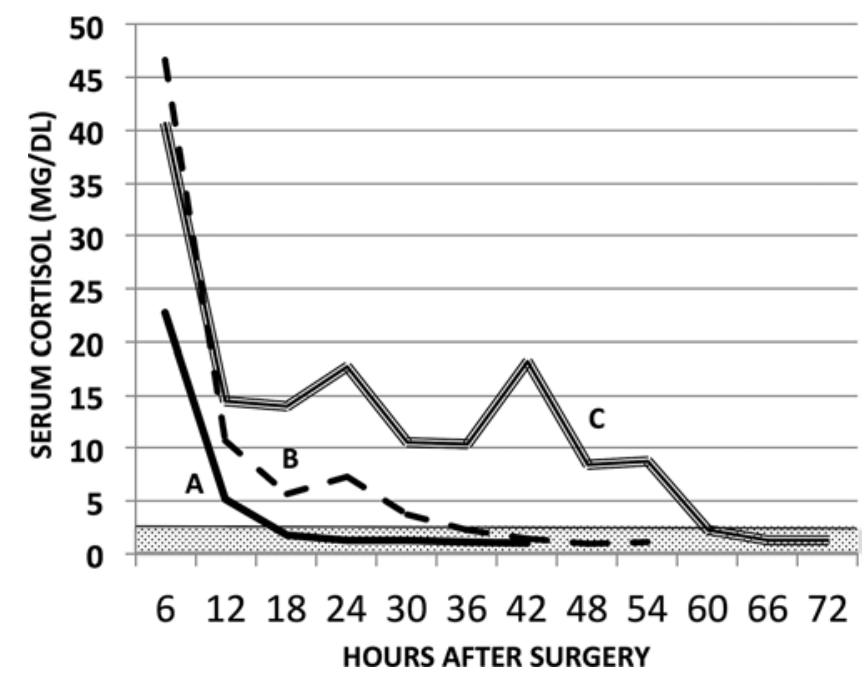

FIG. 4. Individual patient serum cortisol measurements at 6-hour intervals after surgery in 3 representative patients $(A, B$, and $C)$. There is considerable variation in the temporal course of cortisol levels in terms of time to reach a discrimination level of $2.1 \mu \mathrm{g} / \mathrm{dl}$ (stippled area at bottom), i.e., < 24 hours (A), 24-48 hours (B), and 48-72 hours (C). Also noted are occasional transient spikes in cortisol during the period of declining levels (patients B and C).

$\mathrm{dl}$, remission was achieved in $9(75 \%)$ of 12 , including 3 of 3 on POD 1, 4 of 5 on POD 2, and 2 of 4 on POD 3. Two (10\%) of 20 patients with cortisol levels $>5 \mu \mathrm{g} / \mathrm{dl}$ achieved remission. Patients with serum cortisol nadir $<5.4 \mu \mathrm{g} / \mathrm{dl}$ at any time point after surgery had a significantly higher incidence of remission than those with cortisol nadir $>5.4$ $\mu \mathrm{g} / \mathrm{dl}$ (Table 5; $\mathrm{p}<0.001$ ). Furthermore, patients with cortisol nadir $<2.1 \mu \mathrm{g} / \mathrm{dl}$ had a small but significantly higher rate of remission (88.1\%) than patients with cortisol nadir in the range of $2.1-5.4 \mu \mathrm{g} / \mathrm{dl}(75 \%, \mathrm{p}=0.011)$. Patients with postoperative cortisol nadir in the range of $2-5 \mu \mathrm{g} / \mathrm{dl}$ had significantly higher recurrence rates than those with cortisol $<2 \mu \mathrm{g} / \mathrm{dl}(33.3 \%$ vs $5.8 \%, \mathrm{p}=0.010)$.

\section{Discussion}

TSA is the initial treatment of choice for ACTH-secreting pituitary adenomas. ${ }^{18}$ Most patients with CD have microadenomas that can be localized and effectively removed. ${ }^{10}$ Numerous retrospective studies have described postoperative remission rates of $70 \%-98 \%$ for TSA in CD. ${ }^{2,4,5,18} \mathrm{~A}$ number of factors have been identified that contribute to the success or failure of surgery for $\mathrm{CD}$, such as tumor size, prior surgery, visibility of tumor on MRI,

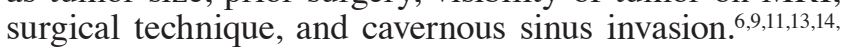
$17,20,27,29,31$ We noted similar findings in the current study, with an overall 6-month remission rate of 78\% and higher remission rates $(88.5 \%$ vs $45 \%)$ noted in patients without prior TSA for CD. Similarly, Dimopoulou et al. ${ }^{9}$ noted remission in only $42 \%$ of patients with prior surgery for CD. We did not observe an association between remission rate and tumor size, most likely related to the limited number of macroadenomas. Additionally, in our study tumors not visible on MRI had remission rates equivalent to those of microadenomas, possibly due to the surgical protocol of
TABLE 4. ROC curve analysis of cortisol measurements according to $P O D$

\begin{tabular}{lccc}
\hline Cortisol Measurement & Cumulative & POD 1 & POD 2 \\
\hline Optimal cortisol value & 2.14 & 5.29 & 5.35 \\
\hline AUC & 0.922 & 0.831 & 0.917 \\
\hline Sensitivity & 0.825 & 0.730 & 0.844 \\
\hline Specificity & 0.812 & 0.875 & 0.933 \\
\hline PPV & 0.942 & 0.956 & 0.965 \\
\hline NPV & 0.555 & 0.466 & 0.731 \\
\hline
\end{tabular}

bilateral exploration and immediate second surgery for elevated postoperative cortisol.

Late recurrence is relatively common in patients who achieve initial remission after surgery for $\mathrm{CD}$, with retrospective analyses reporting recurrent $\mathrm{CD}$ in $7 \%-66 \%$. $^{2,4}$, $5,9,18,30$ These rates vary substantially depending upon tumor characteristics and surgical technique, but appear to be highly dependent upon the duration of follow-up. Recent retrospective analyses of patients with $C D$ and initial remission after surgery followed for a mean of 14 and 16 years found recurrence rates of $66 \%$ and $24 \%$, respectively. ${ }^{2,4}$ We observed late recurrences in $6(9.5 \%)$ of 63 patients at a mean follow-up of 49.3 months among patients with initial remission (Tables 2 and 5). Patients receiving immediate second surgery did not experience a higher incidence of late recurrence (4.3\%) than patients with a single surgery $(10.9 \%)$ or with the overall cohort $(9.2 \%)$, although there was a suggestion of more late recurrence (22\%) in patients with a history of prior surgery for CD. Notably, 2 of 3 patients with late recurrence in the postoperative cortisol $<2 \mu \mathrm{g} / \mathrm{dl}$ group had a history of prior surgery, possibly due to microscopic residual tumor in fibrotic dura. We also noted (Table 5) a higher rate of late recurrence in patients with postoperative cortisol levels in the range of 2.1-5.4 $\mu \mathrm{g} / \mathrm{dl}$ than in those with levels $<2.1 \mu \mathrm{g} / \mathrm{dl}(33.3 \%$ vs $5.8 \%$, $\mathrm{p}=0.01$ ).

Since the initial observation by Ram et al. in 1994, ${ }^{24}$ early repeat surgery based upon normal or supranormal postoperative cortisol levels appears to be an effective strategy to increase the percentage of patients achieving remission after surgery for CD..$^{12,24}$ Importantly, early sur-

TABLE 5. Remission and late recurrence by postoperative serum cortisol nadir category (initial and second surgeries)

\begin{tabular}{lcrccc}
\hline $\begin{array}{c}\text { Cortisol } \\
\text { Nadir } \\
(\mu \mathrm{g} / \mathrm{dl})\end{array}$ & $\begin{array}{c}\text { No. } \\
\text { of Pts }\end{array}$ & $\begin{array}{c}\text { Remission } \\
(\%)\end{array}$ & $\mathrm{p}$ Value & $\begin{array}{c}\text { Late } \\
\text { Recurrence } \\
(\%)\end{array}$ & $\begin{array}{c}\mathrm{p} \\
\text { Value }\end{array}$ \\
\hline$<2.1$ & 59 & $52(88.1)$ & $0.011^{*},<0.001 \dagger$ & $3 / 52(5.8)$ & $0.010 \ddagger$ \\
\hline $2.1-5.4$ & 12 & $9(75.0)$ & & $3 / 9(33.3)$ & \\
\hline$>5.4$ & 20 & $2(10.0)$ & $0 / 2(0)$ & \\
\hline Total & 81 & $63(77.8)$ & $6 / 63(9.5)$ & \\
\hline
\end{tabular}

Pts $=$ patients.

* For comparison between cortisol nadir $<2.1$ versus $2.1-5.4 \mu \mathrm{g} / \mathrm{dl}$ groups. $\dagger$ For comparison between both groups with cortisol nadir $<5.4 \mu \mathrm{g} / \mathrm{dl}$ versus the $>5.4 \mu \mathrm{g} / \mathrm{dl}$ group.

$\ddagger$ For comparison between cortisol nadir $2.1-5.4$ versus $<2.1 \mu \mathrm{g} / \mathrm{dl}$ groups. 
gery is associated with less scarring and inflammation in the gland, enabling a better identification of residual tumor or capsule. In addition, immediate second surgery offers the advantages of a single hospitalization, withholding routine postoperative corticosteroids, and close observation for potential profound postoperative hypocortisolism that occurs after successful surgery. In the current study, remission in $18(78 \%)$ of 23 patients with immediate second surgery was equivalent to the rate for single surgery and for the overall cohort. Presumably few, if any, of the 2-surgery patient cohort with supranormal postoperative cortisol levels after the initial surgery would have achieved remission without a second operation. Excluding 11 patients with postoperative cortisol levels $>5 \mu \mathrm{g} / \mathrm{dl}$ who were not offered a second operation due to history of TSA for $\mathrm{CD}$, the overall remission rate for the 2-surgery protocol was $62(88.6 \%)$ of 70 patients. Immediate second surgery was not associated with a higher incidence of late recurrence, CSF leak, or other surgical complications. However, as previously noted by Ram et al., ${ }^{24}$ patients with immediate second surgery had a significantly elevated risk of hypocortisolism (52\%), which was more than twice the risk of the single-surgery cohort. This is primarily because the second surgery involves more extensive exploration and often partial hypophysectomy. It is important to note, however, that the efficacy and safety of a 2-surgery approach enables a less aggressive resection at the initial operation and may reduce hypocortisolism in single-surgery patients; in fact, the hypocortisolism rate for patients with a single operation in this study was only $22 \%$, which is lower than that of most reported CD surgery series. ${ }^{6,12,18,24,25}$

Suppression of normal pituitary gland corticotroph cells by prolonged exposure to elevated serum ACTH and cortisol in CD produces a characteristic low serum cortisol, which is apparent shortly after successful tumor removal, and can persist for 6-12 months. ${ }^{3,18,20,23,26}$ For both patients in remission and those not in remission, we observed parallel curves in cumulative mean postoperative cortisol levels, with a rapid decline in the first 24 hours to a relatively stable level by $36-72$ hours. Mean cortisol values at every time point were significantly lower in the remission cohort, reaching the subnormal range by $24-30$ hours. However, there was substantial variation among individual patient cortisol time curves, with transient spike elevations in cortisol in both groups and a small number of patients in remission who did not achieve levels $<2 \mu \mathrm{g} / \mathrm{dl}$ until 48-72 hours after surgery. Patients who achieved levels $<2 \mu \mathrm{g} / \mathrm{dl}$ at any day were equally likely to achieve remission. In the current study, no variables could be identified that correlated with the temporal dynamics of postoperative cortisol. However, we were unable to measure the duration and severity of $\mathrm{CD}$, which may have contributed to the degree of postoperative corticotroph suppression or the existence of Crooke's cell changes. In addition, Monteith et al. ${ }^{20}$ showed that rapid decline in postoperative cortisol after surgery for $\mathrm{CD}$ was significantly associated with extracapsular en bloc tumor resection; we attempted this technique in all initial surgeries but did not record successful extracapsular resection from the surgical record. Importantly, these observations suggest that single measurements showing elevated serum cortisol in the first 60 hours after surgery for $C D$ may be inaccurate in predicting remission due to a delayed temporal decline or a transient spike, and that these temporal profiles cannot be predicted from preoperative clinical or imaging features.

Several retrospective analyses have described a higher likelihood of remission or a lower rate of recurrence after surgery for CD if the serum cortisol or ACTH levels were subnormal, although the timing of cortisol sampling varied considerably. 1,3,5-7,12,13,15,16,19-22,24,26,28-30,32-34 Sughrue et al ${ }^{30}$ performed a systematic review and fixed-effects meta-analysis of postoperative cortisol measurement and risk of recurrence after surgery for $\mathrm{CD}$. They found cumulative recurrence rates of $9 \%$ and $24 \%$ for subnormal versus normal postoperative cortisol, respectively, which corresponded to their own retrospective experience. However, there was considerable variation among the 15 studies analyzed in the timing of cortisol determination (1-28 days), definition of subnormal cortisol $(2-11 \mu \mathrm{g} / \mathrm{dl})$, definition of recurrence (morning cortisol vs 24-hour UFC), duration of follow-up (24-115 months), and use of supplemental corticosteroids in the postoperative period. With the objective of offering immediate second surgery to patients otherwise unlikely to achieve remission, it is important to know the optimal timing and most accurate discriminating value to predict remission from postoperative cortisol determinations. In this study we followed a strict protocol of measuring postoperative cortisol levels every 6 hours for the first 72 hours after surgery, and recommended a repeat operation for patients not achieving a cortisol nadir of $<2 \mu \mathrm{g} /$ dl by 72 hours. When combining the data for all patients, ROC curve analysis showed an optimal discriminatory level of $2.1 \mu \mathrm{g} / \mathrm{dl}$, which produced a PPV of $94 \%$, although the NPV was lower (55\%). These values correspond to an analogous study by Costenaro et $a .^{8}$ in which a cortisol nadir $\leq 3.5 \mu \mathrm{g} / \mathrm{dl}$ within 48 hours of surgery had a PPV of $100 \%$ and NPV of $60 \%$. In a practical sense, patients with postoperative cortisol $<2.1 \mu \mathrm{g} / \mathrm{dl}$ have a very high likelihood of remission (although not 100\%). Conversely, recommending repeat surgery for all patients with values $>2.1 \mu \mathrm{g} / \mathrm{dl}$ would subject a small percentage of patients in remission to unnecessary surgery. Using a discrimination level of $5.4 \mu \mathrm{g} / \mathrm{dl}$ at 48 hours after surgery resulted in an equivalent PPV (96\%) and a better NPV (73\%). When applying these discrimination levels post hoc to our cumulative data (Table 4), a postoperative nadir cortisol level $<5.4$ $\mu \mathrm{g} / \mathrm{dl}$ was highly significantly associated with remission. However, the subgroup of patients with postoperative cortisol nadir in the range of $2.1-5.4 \mu \mathrm{g} / \mathrm{dl}$ had a significantly lower remission rate $(75 \%)$ versus those with levels $<2 \mu \mathrm{g} /$ dl $(88 \%)$. Thus, immediate second surgery should be offered to patients with cortisol levels $>5.4 \mu \mathrm{g} / \mathrm{dl}$ by 72 hours after surgery. Patients with postoperative serum cortisol levels $<2.1 \mu \mathrm{g} / \mathrm{dl}$ within 72 hours do not require second surgery, although a small number will not achieve remission. Patients in the intermediate range $(2.1-5.4 \mu \mathrm{g} / \mathrm{dl})$ have a slightly lower remission rate, but nevertheless have an excellent chance of remission without a second operation.

There are inherent limitations in the single-center retrospective nature of this study, including potential selection and referral bias, differential losses to follow-up, and limitations of retrospective data quality. As above, the details 
of tumor removal (i.e., en bloc vs piecemeal resection) were not recorded and noncortisol pituitary hormone status after surgery was not analyzed. On the other hand, patients were diagnosed with $\mathrm{CD}$ and selected for surgery according to standardized protocols, and data for this analysis were derived from a prospectively acquired database of all patients undergoing pituitary surgery. This was particularly important for the postoperative cortisol data, which to our knowledge is the most comprehensive assessment of this variable at consistent time intervals over multiple days. Some patients with a history of prior surgery for CD and normal or supranormal postoperative cortisol levels after single surgery were not offered second surgery due to the likelihood of residual tumor in areas of dural fibrosis; it is possible that some proportion of this cohort may have achieved remission with 2 surgeries. Although the cohort size limited statistical analysis of subgroups, the study represents one of the largest and most comprehensive single-institution analyses of the predictive value of postoperative serum cortisol determinations after surgery for CD. Although patient follow-up was thorough according to the center protocols, there may have been late recurrences that were lost to follow-up. Because late recurrences of CD appear to continue over long intervals, ${ }^{2,9,25}$ the mean follow-up of 49 months likely led to an underestimation of late recurrence rates. Absent data regarding the duration and severity of CD prior to surgery and its effect upon the degree of normal gland inhibition ${ }^{23}$ may be an unrecognized variable in the temporal dynamics of postoperative cortisol.

\section{Conclusions}

The temporal course of serum cortisol levels after surgery for $\mathrm{CD}$ is somewhat variable, and is not predicted by any apparent preoperative clinical or imaging features. Postoperative serum cortisol within 72 hours can predict subsequent remission with excellent accuracy. Immediate second surgery using postoperative cortisol criteria is highly effective in improving remission rates, and is safe with the exception of a higher incidence of hypocortisolism. The safety and efficacy of a second operation based on postoperative cortisol enables a less aggressive initial operation, and therefore may lower the overall rate of hypocortisolism for the entire cohort. Treatment and management of CD at any stage of care requires coordination with a multidisciplinary team and treatment protocols. Lastly, postoperative cortisol monitoring and immediate second surgery is likely to be cost-effective by reducing readmission for delayed second surgery, and for the benefits of improved long-term remission rates with lower attendant health care expenditures.

\section{Acknowledgments}

We thank Dr. Uresh Patel for his independent interpretation of the MR images.

\section{References}

1. Abellán Galiana P, Fajardo Montañana C, Riesgo Suárez PA, Gómez Vela J, Escrivá CM, Lillo VR: [Predictors of longterm remission after transsphenoidal surgery in Cushing's disease.] Endocrinol Nutr 60:475-482, 2013 (Span)

2. Alexandraki KI, Kaltsas GA, Isidori AM, Storr HL, Afshar
F, Sabin I, et al: Long-term remission and recurrence rates in Cushing's disease: predictive factors in a single-centre study. Eur J Endocrinol 168:639-648, 2013

3. Alwani RA, de Herder WW, de Jong FH, Lamberts SW, van der Lely AJ, Feelders RA: Rapid decrease in adrenal responsiveness to ACTH stimulation after successful pituitary surgery in patients with Cushing's disease. Clin Endocrinol (Oxf) 75:602-607, 2011

4. Aranda G, Enseñat J, Mora M, Puig-Domingo M, Martínez de Osaba MJ, Casals G, et al: Long-term remission and recurrence rate in a cohort of Cushing's disease: the need for long-term follow-up. Pituitary 18:142-149, 2015

5. Barbetta L, Dall'Asta C, Tomei G, Locatelli M, Giovanelli M, Ambrosi B: Assessment of cure and recurrence after pituitary surgery for Cushing's disease. Acta Neurochir (Wien) 143:477-482, 2001

6. Blevins LS Jr, Christy JH, Khajavi M, Tindall GT: Outcomes of therapy for Cushing's disease due to adrenocorticotropinsecreting pituitary macroadenomas. J Clin Endocrinol Metab 83:63-67, 1998

7. Bochicchio D, Losa M, Buchfelder M: Factors influencing the immediate and late outcome of Cushing's disease treated by transsphenoidal surgery: a retrospective study by the European Cushing's Disease Survey Group. J Clin Endocrinol Metab 80:3114-3120, 1995

8. Costenaro F, Rodrigues TC, Rollin GA, Ferreira NP, Czepielewski MA: Evaluation of Cushing's disease remission after transsphenoidal surgery based on early serum cortisol dynamics. Clin Endocrinol (Oxf) 80:411-418, 2014

9. Dimopoulou C, Schopohl J, Rachinger W, Buchfelder M, Honegger J, Reincke M, et al: Long-term remission and recurrence rates after first and second transsphenoidal surgery for Cushing's disease: care reality in the Munich Metropolitan Region. Eur J Endocrinol 170:283-292, 2013

10. Doppman JL, Frank JA, Dwyer AJ, Oldfield EH, Miller DL, Nieman LK, et al: Gadolinium DTPA enhanced MR imaging of ACTH-secreting microadenomas of the pituitary gland. J Comput Assist Tomogr 12:728-735, 1988

11. Feng M, Bao X, Lu L, Deng K, Liu X, Dai C, et al: [Diagnosis and surgical management in patients with Cushing disease with negative magnetic resonance imaging.] Zhonghua Yi Xue Za Zhi 95:3213-3216, 2015 (Chinese)

12. Hameed N, Yedinak CG, Brzana J, Gultekin SH, Coppa ND, Dogan A, et al: Remission rate after transsphenoidal surgery in patients with pathologically confirmed Cushing's disease, the role of cortisol, ACTH assessment and immediate reoperation: a large single center experience. Pituitary 16:452-458, 2013

13. Hanson JM, Teske E, Voorhout G, Galac S, Kooistra HS, Meij BP: Prognostic factors for outcome after transsphenoidal hypophysectomy in dogs with pituitary-dependent hyperadrenocorticism. J Neurosurg 107:830-840, 2007

14. Knosp E, Steiner E, Kitz K, Matula C: Pituitary adenomas with invasion of the cavernous sinus space: a magnetic resonance imaging classification compared with surgical findings. Neurosurgery 33:610-618, 1993

15. Krikorian A, Abdelmannan D, Selman WR, Arafah BM: Cushing disease: use of perioperative serum cortisol measurements in early determination of success following pituitary surgery. Neurosurg Focus 23(3):E6, 2007

16. Lindsay JR, Oldfield EH, Stratakis CA, Nieman LK: The postoperative basal cortisol and CRH tests for prediction of long-term remission from Cushing's disease after transsphenoidal surgery. J Clin Endocrinol Metab 96:2057-2064, 2011

17. Lonser RR, Ksendzovsky A, Wind JJ, Vortmeyer AO, Oldfield EH: Prospective evaluation of the characteristics and incidence of adenoma-associated dural invasion in Cushing disease. J Neurosurg 116:272-279, 2012

18. Lonser RR, Nieman L, Oldfield EH: Cushing's disease: 
pathobiology, diagnosis, and management. J Neurosurg 126:404-417, 2017

19. McCance DR, Gordon DS, Fannin TF, Hadden DR, Kennedy L, Sheridan B, et al: Assessment of endocrine function after transsphenoidal surgery for Cushing's disease. Clin Endocrinol (Oxf) 38:79-86, 1993

20. Monteith SJ, Starke RM, Jane JA Jr, Oldfield EH: Use of the histological pseudocapsule in surgery for Cushing disease: rapid postoperative cortisol decline predicting complete tumor resection. J Neurosurg 116:721-727, 2012

21. Pendharkar AV, Sussman ES, Ho AL, Hayden Gephart MG, Katznelson L: Cushing's disease: predicting long-term remission after surgical treatment. Neurosurg Focus 38(2):E13, 2015

22. Pereira AM, van Aken MO, van Dulken H, Schutte PJ, Biermasz NR, Smit JW, et al: Long-term predictive value of postsurgical cortisol concentrations for cure and risk of recurrence in Cushing's disease. J Clin Endocrinol Metab 88:5858-5864, 2003

23. Raff H, Carroll T: Cushing's syndrome: from physiological principles to diagnosis and clinical care. J Physiol 593:493506,2015

24. Ram Z, Nieman LK, Cutler GB Jr, Chrousos GP, Doppman JL, Oldfield EH: Early repeat surgery for persistent Cushing's disease. J Neurosurg 80:37-45, 1994

25. Rees DA, Hanna FW, Davies JS, Mills RG, Vafidis J, Scanlon MF: Long-term follow-up results of transsphenoidal surgery for Cushing's disease in a single centre using strict criteria for remission. Clin Endocrinol (Oxf) 56:541-551, 2002

26. Rollin GA, Ferreira NP, Junges M, Gross JL, Czepielewski MA: Dynamics of serum cortisol levels after transsphenoidal surgery in a cohort of patients with Cushing's disease. J Clin Endocrinol Metab 89:1131-1139, 2004

27. Santoro A, Minniti G, Ruggeri A, Esposito V, Jaffrain-Rea ML, Delfini R: Biochemical remission and recurrence rate of secreting pituitary adenomas after transsphenoidal adenomectomy: long-term endocrinologic follow-up results. Surg Neurol 68:513-518, 2007

28. Srinivasan L, Laws ER, Dodd RL, Monita MM, Tannenbaum CE, Kirkeby KM, et al: The dynamics of post-operative plasma ACTH values following transsphenoidal surgery for Cushing's disease. Pituitary 14:312-317, 2011

29. Starke RM, Reames DL, Chen CJ, Laws ER, Jane JA Jr: Endoscopic transsphenoidal surgery for cushing disease: techniques, outcomes, and predictors of remission. Neurosurgery 72:240-247, 2013

30. Sughrue ME, Shah JK, Devin JK, Kunwar S, Blevins LS Jr: Utility of the immediate postoperative cortisol concentrations in patients with Cushing's disease. Neurosurgery 67:688695,2010
31. Sun Y, Sun Q, Fan C, Shen J, Zhao W, Guo Y, et al: Diagnosis and therapy for Cushing's disease with negative dynamic MRI finding: a single-centre experience. Clin Endocrinol (Oxf) 76:868-876, 2012

32. Trainer PJ, Lawrie HS, Verhelst J, Howlett TA, Lowe DG, Grossman AB, et al: Transsphenoidal resection in Cushing's disease: undetectable serum cortisol as the definition of successful treatment. Clin Endocrinol (Oxf) 38:73-78, 1993

33. van Aken MO, de Herder WW, van der Lely AJ, de Jong FH, Lamberts SW: Postoperative metyrapone test in the early assessment of outcome of pituitary surgery for Cushing's disease. Clin Endocrinol (Oxf) 47:145-149, 1997

34. Yap LB, Turner HE, Adams CB, Wass JA: Undetectable postoperative cortisol does not always predict long-term remission in Cushing's disease: a single centre audit. Clin Endocrinol (Oxf) 56:25-31, 2002

\section{Disclosures}

The authors report no conflict of interest concerning the materials or methods used in this study or the findings specified in this paper.

\section{Author Contributions}

Conception and design: Mayberg, Moloney, Mercado, Scanlan, Broyles. Acquisition of data: Mayberg, Reintjes, Patel, Moloney, Mercado, Scanlan, Broyles. Analysis and interpretation of data: all authors. Drafting the article: Mayberg, Reintjes, Patel, Carlson, Scanlan. Critically revising the article: Mayberg, Reintjes, Patel, Moloney, Mercado, Carlson, Scanlan. Reviewed submitted version of manuscript: all authors. Approved the final version of the manuscript on behalf of all authors: Mayberg. Statistical analysis: Mayberg, Carlson, Scanlan. Administrative/technical/material support: Mayberg.

\section{Supplemental Information}

\section{Previous Presentations}

Portions of this work were presented in abstract form at the American Academy of Neurological Surgery meeting, Jackson Hole, WY, September 14, 2016.

\section{Correspondence}

Marc R. Mayberg, Department of Neurological Surgery, University of Washington, UW Medicine Neuroscience Institute, 325 Ninth Ave., Box 359766, Seattle, WA 98104. email: maybergm@ uw.edu. 